AN

INTRODUCTION

TO GAUGE

THEORIES

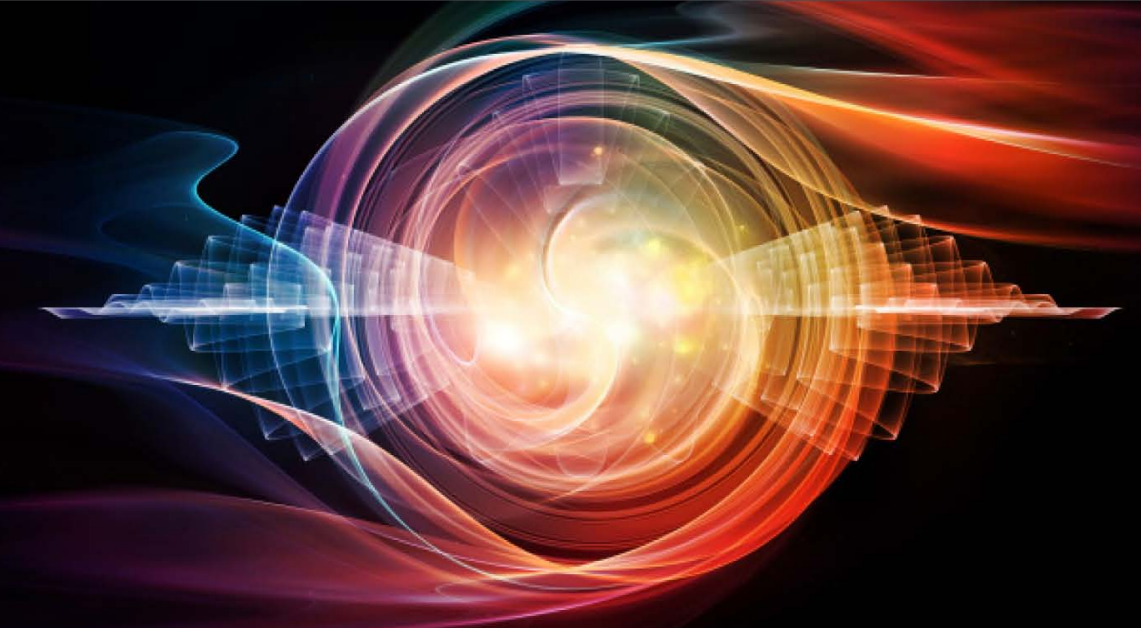

Nicola Cabibibo

Luciano Maiani

Omar Benhar

CRC $\underset{\text { Taylor \& Francis Group }}{\text { CRC Press }}$ 


\section{AN INTRODUCTION \\ TO GAUGE THEORIES}




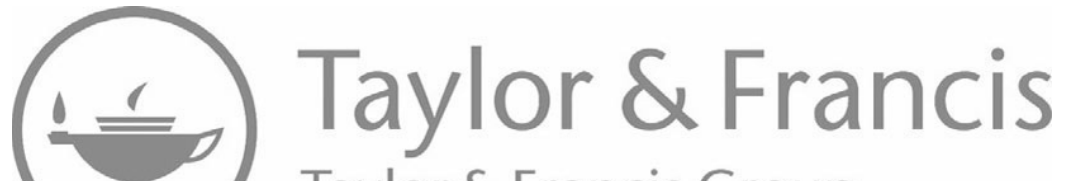
Taylor \& Francis Group http://taylorandfrancis.com 


\section{AN INTRODUCTION TO GAUGE THEORIES}

\section{Nicola Cabibbo}

University of Rome, La Sapienza, and

INFN Sezione di Roma, Rome

\section{Luciano Maiani}

University of Rome, La Sapienza, and

INFW Sezione di Roma, Rome

Omar Benhar

INFW Sezione di Roma, and

University of Rome, La Sapienza

Iranslated from the original Italian by Geoffrey Hall, Imperial College, London

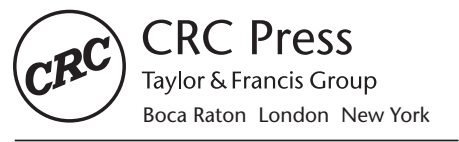

CRC Press is an imprint of the

Taylor \& Francis Group, an informa business 
This book was first published in Italian in 2016, by Editori Riuniti University Press, under the original title: Introduzione alle Teorie di Gauge.

\section{CRC Press}

Taylor \& Francis Group

6000 Broken Sound Parkway NW, Suite 300

Boca Raton, FL 33487-2742

(C) 2018 by Taylor \& Francis Group, LLC

CRC Press is an imprint of Taylor \& Francis Group, an Informa business

No claim to original U.S. Government works

Printed on acid-free paper

International Standard Book Number-13: 978-1-4987-3451-6 (Hardback)

This book contains information obtained from authentic and highly regarded sources. Reasonable efforts have been made to publish reliable data and information, but the author and publisher cannot assume responsibility for the validity of all materials or the consequences of their use. The authors and publishers have attempted to trace the copyright holders of all material reproduced in this publication and apologize to copyright holders if permission to publish in this form has not been obtained. If any copyright material has not been acknowledged please write and let us know so we may rectify in any future reprint.

Except as permitted under U.S. Copyright Law, no part of this book may be reprinted, reproduced, transmitted, or utilized in any form by any electronic, mechanical, or other means, now known or hereafter invented, including photocopying, microfilming, and recording, or in any information storage or retrieval system, without written permission from the publishers.

For permission to photocopy or use material electronically from this work, please access www.copyright.com (http://www.copyright.com/) or contact the Copyright Clearance Center, Inc. (CCC), 222 Rosewood Drive, Danvers, MA 01923, 978-750-8400. CCC is a not-for-profit organization that provides licenses and registration for a variety of users. For organizations that have been granted a photocopy license by the CCC, a separate system of payment has been arranged.

Trademark Notice: Product or corporate names may be trademarks or registered trademarks, and are used only for identification and explanation without intent to infringe.

\section{Library of Congress Cataloging-in-Publication Data}

Names: Cabibbo, N., author. | Maiani, L. (Luciano), author. | Benhar, Omar, author.

Title: Introduction to gauge theories / Nicola Cabibbo, Luciano Maiani, Omar Benhar.

Description: Boca Raton, FL : CRC Press, Taylor \& Francis Group, [2017] |

Includes bibliographical references and index.

Identifiers: LCCN 2016041647| ISBN 9781498734516 (hardback ; alk. paper) |

ISBN 9781498734516 (E-book).

Subjects: LCSH: Gauge fields (Physics) | Field theory (Physics).

Classification: LCC QC793.3.G38 C33 2017 | DDC 530.14/35--dc23.

LC record available at https://lccn.loc.gov/2016041647

\section{Visit the Taylor \& Francis Web site at}

http://www.taylorandfrancis.com

and the CRC Press Web site at

http://www.crcpress.com 


\section{Contents}

List of Figures $\quad$ xi

Preface xiii

The Authors $\quad$ XV

\begin{tabular}{ccr} 
ChAPTER & $1 \cdot$ INTRODUCTION & 1 \\
\hline $1.1 \quad$ QUANTUM ELECTRODYNAMICS & 1
\end{tabular}

1.2 UNITS AND OTHER CONVENTIONS. 3

CHAPTER 2 - THE FEYNMAN PATH INTEGRAL 5

2.1 CALCULATION OF THE TRANSITION AMPLITUDE 5

2.2 THE LATTICE APPROXIMATION 8

2.3 THE CLASSICAL LIMIT 9

2.4 TIME AS A COMPLEX VARIABLE 9

2.5 STATISTICAL MECHANICS

2.6 GREEN'S FUNCTIONS 12

\begin{tabular}{crr} 
ChAPTER & 3 - TOWARDS A FIELD THEORY & 17 \\
\hline $3.1 \quad$ THE GENERATING FUNCTIONAL & 20
\end{tabular}

3.2 THE HARMONIC OSCILLATOR 23

3.3 FREE SCALAR FIELDS: PROPAGATOR AND GENERATING FUNCTIONAL 28

3.4 FREE SCALAR FIELD: ONE-PARTICLE STATES 31

3.5 CREATION AND DESTRUCTION OPERATORS 33

Chapter 4 - EQUATIONS OF MOTION, SYMMETRIES AND WARD'S IDENTITY $\quad 37$

4.1 SUM OVER PATHS AND OPERATORS 38

4.1.1 Derivatives 
4.2 THE FUNDAMENTAL IDENTITY 39

4.3 QUANTUM MECHANICS 41

4.3.1 Equations of motion and commutation rules 41

4.3.2 Symmetries 43

4.3.3 The Hamiltonian function 45

4.4 FIELD THEORY 46

4.4.1 Symmetries in field theory $\quad 47$

4.4.2 Ward's identity 49

4.5 THE SYMMETRIES OF THE VACUUM 52

Chapter 5 - THE ELECTROMAGNETIC FIELD 55

5.1 THE CHOICE OF GAUGE 56

5.2 GENERATING FUNCTIONAL AND PROPAGATOR 58

5.3 SINGLE PHOTON STATES 59

5.4 VIRTUAL PHOTONS 62

\begin{tabular}{lll} 
CHAPTER $\quad 6 \cdot$ FERMION FIELDS & 67 \\
\hline
\end{tabular}

6.1 HARMONIC AND FERMI OSCILLATORS 67

6.1.1 Anticommuting variables 69

6.1.2 Sum over paths for the two oscillators $\quad 70$

6.1.3 Gaussian integrals for commuting and anticommuting variables 74

6.2 QUANTISATION OF THE DIRAC FIELD 76

$\begin{array}{lll}6.2 .1 & \text { Fermion propagator } & 79\end{array}$

$\begin{array}{lll}\text { 6.2.2 The spin-statistics theorem } & 79\end{array}$

6.2.3 One-particle states of the Dirac field 81

\begin{tabular}{ll} 
CHAPTER & 7 - SCATTERING PROCESSES AND THE $S$-MATRIX 85 \\
\hline 7.1 & SCIN"STATESAND "OUT"STATES
\end{tabular}

7.1 "IN" STATES AND "OUT" STATES 86

7.2 SCATTERING AMPLITUDES AND THE $S$-MATRIX 89

7.3 CONSERVED QUANTITIES 90

7.4 THE LSZ REDUCTION FORMULAE 91

\begin{tabular}{lll} 
CHAPTER $\quad 8$ - PERTURBATIVE GREEN'S FUNCTIONS IN $\lambda \phi^{4} \quad 99$ \\
\hline 8.1
\end{tabular}

8.1 THE PERTURBATIVE GENERATING FUNCTIONAL 100

8.2 FEYNMAN RULES FOR GREEN'S FUNCTIONS 104 
8.3 CONNECTED PARTS AND VACUUM DIAGRAMS

8.4 PERTURBATIVE TWO-POINT GREEN'S FUNCTION

\begin{tabular}{lll} 
CHAPTER & $9 \cdot S$-MATRIX FEYNMAN DIAGRAMS IN $\lambda \phi^{4} \quad 117$ \\
\hline 9.1 & ONE-PARTICLEER
\end{tabular}

9.1 ONE-PARTICLE IRREDUCIBLE DIAGRAMS

9.2 FEYNMAN RULES FOR THE $S$-MATRIX ELEMENTS

10.1 FEYNMAN DIAGRAMS FOR THE GENERATING FUNCTIONAL

10.2 TWO-POINT FUNCTIONS

10.3 REDUCTION FORMULAE

10.4 FEYNMAN DIAGRAMS FOR THE $S$-MATRIX

10.5 COMBINATORIALS

11.1 THE PHOTON PROPAGATOR

11.2 RENORMALISATION OF THE CHARGE

11.3 THE ELECTRON PROPAGATOR

11.3.1 The propagator to all orders

11.4 THE VERTEX

11.5 WARD'S IDENTITY

\begin{tabular}{lll} 
ChAPTER 12 - APPLICATIONS OF QED & 155 \\
\hline
\end{tabular}

12.1 SCATTERING IN AN EXTERNAL FIELD

12.2 BREMSSTRAHLUNG AND INFRARED DIVERGENCE 158

12.3 THE LAMB SHIFT 162

12.4 VACUUM POLARISATION 166

12.4.1 Calculation of the tensor $\Pi^{\mu \nu}(k)$ to one loop 166

12.5 THE ANOMALOUS MAGNETIC MOMENT 169

12.5.1 Preliminaries 169

$\begin{array}{ll}\text { 12.5.2 The calculation } & 171\end{array}$

\begin{tabular}{lll} 
CHAPTER & 13 - RENORMALISATION GROUP OF QED & 175 \\
\hline
\end{tabular}

13.1 EFFECTIVE ELECTRIC CHARGE 176

13.2 THE GELL-MANN AND LOW EQUATION 179 
13.3 THE QED $\beta$ FUNCTION

13.4 ASYMPTOTIC VARIATION OF THE EFFECTIVE CHARGE 181

CHAPTER 14 - QUANTISING A NON-ABELIAN THEORY

14.1 FUNDAMENTALS

14.2 QUARKS IN QUANTUM CHROMODYNAMICS

14.3 THE FADDEEV-POPOV DETERMINANT

14.4 FEYNMAN RULES

ChAPTER 15 - THE $\beta$ FUNCTION IN QCD

195

15.1 VACUUM POLARISATION

15.2 CORRECTIONS TO QUARK PROPAGATOR AND VERTEX 200

15.3 ASYMPTOTIC FREEDOM

CHAPTER 16 - UNITARITY AND GHOSTS 205

16.1 THE CUTKOSKY RULE 206

16.2 THE INELASTIC REACTION $u+\bar{u} \rightarrow d+\bar{d}$

16.3 THE CASE OF QED

16.4 NON-ABELIAN GAUGE THEORIES

ChAPTER 17 - EFFECTIVE CONSTANTS AT HIGH ENERGY AND GRAND UNIFICATION

17.1 THE DETERMINATION OF $\alpha_{s}$

17.2 THE LANDAU POLE AND THE CONTINUUM LIMIT

17.3 EFFECTIVE CONSTANTS OF THE STANDARD THEORY

17.4 GRAND UNIFICATION AND OTHER HYPOTHESES

ChAPTER 18 - LIMITS ON THE MASS OF THE HIGGS BOSON 229

18.1 SCALAR FIELDS IN THE STANDARD THEORY

18.2 LIMITS ON THE MASS OF THE HIGGS BOSON

CHAPTER 19 - THE WEAK MUON ANOMALY

19.1 THE $R_{\xi}$ GAUGE

19.2 MUON ANOMALY: W EXCHANGE

$19.3 Z$ AND HIGGS BOSON EXCHANGE 
20.1 EFFECTIVE POTENTIAL

20.2 EXPANSION AROUND THE CLASSICAL LIMIT

20.3 LOOP EXPANSION OF THE POTENTIAL

20.4 ONE LOOP POTENTIAL IN THE STANDARD THEORY 258

20.5 NON-NATURALNESS OF THE STANDARD THEORY 264

APPENDIX A - Transition Amplitude Calculation 269

A.1 TRANSITION AMPLITUDE FOR ZERO POTENTIAL

APPENDIX B - Connected Diagrams 271

B.1 GENERATING FUNCTIONAL OF CONNECTED DIAGRAMS

APPENDIX $\quad \mathrm{C} \cdot$ Lorentz invariance and one-particle states 275

C.1 RENORMALISATION CONSTANTS

APPENDIX D - Reduction formulae 279

D.1 REDUCTION FORMULAE FOR THE COMPTON SCATTERING AMPLITUDE

APPENDIX E $\quad$ Integrals

283

E.1 INTEGRATION IN $D$ DIMENSIONS

283

E.2 FEYNMAN PARAMETERS

APPENDIX $\quad \mathbf{F} \cdot \beta(\lambda)$ and $\beta\left(g_{t}\right)$ functions

F.1 CALCULATION OF THE $\beta(\lambda)$ AND $\beta\left(g_{t}\right)$ FUNCTIONS 287

F.2 $\beta(\lambda)$

F.3 $\beta\left(g_{t}\right)$

Bibliography

Index 


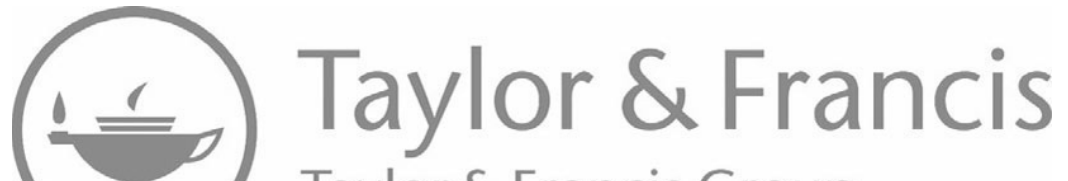
Taylor \& Francis Group http://taylorandfrancis.com 


\section{List of Figures}

$1.1 \quad$ Fundamental fermions.

3.1 Poles and integration paths of the function $G(x, \chi)$.

4.1 Feynman diagrams for Green's function.

5.1 A schematic illustration of gauge trajectories. 56

5.2 Feynman diagram for exchange of a virtual photon. 63

8.1 A graphical representation of the contribution of vertices. 101

8.2 First order diagrams and lowest order two-point function 102

$\begin{array}{lll}8.3 & \text { Propagator corrections to second perturbative order. } & 103\end{array}$

$\begin{array}{lll}8.4 & \text { Vertex corrections to second perturbative order. } & 104\end{array}$

8.5 First-order diagrams for the two-point Green's function. 107

8.6 First-order diagrams for the four-point Green's function. 108

$\begin{array}{lll}8.7 & \text { A disconnected diagram to second order in } \lambda . & 111\end{array}$

8.8 The diagram of order $\lambda$ for the four-point Green's function. 113

$\begin{array}{ll}\text { 8.9 Connected diagrams for the two-point function. } & 114\end{array}$

9.1 Connected diagrams for the four-point Green's function. 118

9.2 A connected diagram one-particle reducible on two external lines. 118

9.3 Block representation of the connected four-point Green's function. 119

9.4 Expansion of the connected, 1PI, four-point Green's function. 119

$\begin{array}{ll}\text { 10.1 The two diagrams with a single vertex. } & 126\end{array}$

10.2 Perturbative diagrams with vertices along open fermion lines. 135

11.1 The photon propagator to order $\alpha$. 142

$\begin{array}{ll}11.2 & \text { Corrections of order } \alpha \text { to the electron propagator. } \\ 146\end{array}$

$\begin{array}{lll}11.3 & \text { Corrections to the electron propagator with two insertions. } & 149\end{array}$ 


\section{xii $\square$ LIST OF FIGURES}

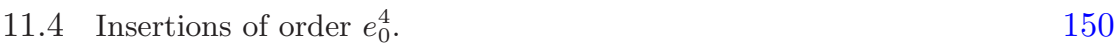

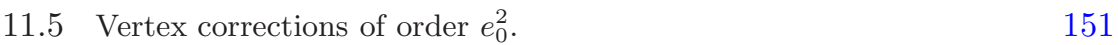

12.1 Feynman diagram for electron scattering in an external field. $\quad 157$

$\begin{array}{lll}12.2 & \text { Feynman diagrams for bremsstrahlung processes. } & 159\end{array}$

$\begin{array}{lll}12.3 & \text { Feynman diagram for electron scattering vertex correction. } & 161\end{array}$

13.1 QED theory compared to experiment for Bhabha scattering at LEP. 178

14.1 Gauge trajectories in the space of the functions $A^{\mu}(x) . \quad 188$

14.2 Feynman diagrams of a Yang-Mills theory with exact symmetry. 193

$\begin{array}{lll}15.1 & \text { Vacuum polarisation corrections in QCD. } & 196\end{array}$

15.2 Renormalisation of the propagator and vertex in QCD. 200

$16.1 d \bar{d}$ contribution to the unitarity relation in $u \bar{u} \rightarrow u \bar{u}$ scattering. 208

16.2 Diagrams showing possible photon exchanges between fermions. 211

16.3 Two gluon intermediate states in fermion-antifermion annihilation. 213

16.4 Inclusion of intermediate ghost state to satisfy unitarity. 215

17.1 Comparison of experimental and theoretical variation of QCD $\alpha_{s} . \quad 219$

17.2 High-energy behaviour of the effective constants, $\alpha, \alpha_{W}$ and $\alpha_{s} . \quad 223$

17.3 Baryon number non-conservation in $S U(5)$ grand unification. 226

17.4 Approximate coupling constant convergence at extreme energy. 228

18.1 $W$ exchange in $b+t \rightarrow t+b$. 233

18.2 Standard Theory behaviour of $\lambda(q)$ to leading log approximation. 236

18.3 Standard Theory behaviour of $\lambda(q)$ to NNLL approximation. 237

19.1 Tree-level diagrams involving vector and scalar propagators. 243

19.2 Charged boson exchange diagrams for the weak muon anomaly. 244

19.3 Neutral boson exchange diagrams for the weak muon anomaly. 247

20.1 Qualitative behaviour of the effective potential at high fields. 264

$\begin{array}{lll}\text { E.1 Wick rotation. } & 284\end{array}$

F.1 Feynman diagrams for the $\beta(\lambda)$ function in the Standard Theory. 288

F.2 Scalar-vector vertex in the Landau gauge. 289

F.3 Feynman diagrams for the $\beta\left(g_{t}\right)$ function in the Standard Theory. 294 


\section{Preface}

Introduction to Gauge Theories completes the series of three volumes based on lecture courses in relativistic quantum mechanics, electroweak interactions and gauge theories, taught by the authors to first-year master's degree students in physics and astronomy, and astrophysics (Laurea Magistrale) of the University of Rome, "La Sapienza", over a period of several decades.

The principal objective of this volume is to introduce the basic concepts of renormalisation in quantum field theory and the fundamentals of modern gauge theories. Even though it is connected to the previous volumes, the book can be read independently; it assumes only a general familiarity with special relativity, second quantisation and the phenomenology of electroweak interactions.

The basic tool is the Feynman path integral, introduced in the early chapters and systematically employed in what follows. The exposition follows a pedagogic course, which begins with the simple case of the quantum mechanical transition amplitude to lead to the generating functional of the Green's functions of field theory. The same pedagogic approach is used in the chapter in which equations of motion, symmetries and the Ward identity are discussed. The analysis of the path integral formalism is completed by a discussion of anticommuting variables and the quantisation of fermion and electromagnetic fields.

The perturbative expansion of the generating functional of the Green's functions, and the Lehman, Symanzyk and Zimmermann reduction formulae - which allow the latter to be related to the scattering matrix elements - are illustrated, first in the simple case of a scalar field theory with $\lambda \phi^{4}$ interactions and subsequently with quantum electrodynamics (QED).

Renormalisation in QED is treated in the central part of the book. The appearance of ultraviolet divergences, from which the necessity of introducing a regularisation procedure follows, is illustrated by taking as examples corrections of order $\alpha$ to the photon and electron propagators and to the interaction vertex. The fundamental Ward identity of QED is also proven to the same perturbative order.

The discussion of QED to second order is supplemented by the detailed illustration of several important applications, which include analysis of the infrared divergence, detailed calculations of the Lamb shift and the vacuum polarisation tensor, and Schwinger's calculation of the correction to the anomalous magnetic moment of the electron, extended in a subsequent chapter to 
the full Electroweak corrections in the Standard Theory. Introduction to the renormalisation group completes the part dedicated to QED, with discussion of the Gell-Mann and Low equation, and the Landau pole.

In the following part of the book, quantisation of non-Abelian gauge theories is analysed, and the evolution of the coupling constant with the energy and momentum scale. Asymptotic freedom in quantum chromodynamics, the fundamental theory of strong interactions, is demonstrated to second perturbative order through explicit calculation of the $\beta$ function. The evolution of the coupling constant of the fundamental interactions to high and very high energies is discussed as an introduction to the hypothesis of grand unification.

The recent discovery of the Higgs boson has reopened discussion of the naturalness, or non-naturalness, of scalar fields in field theory, also in connection with the possible presence of new physics at energies higher than the critical energy of the Standard Theory. The final chapters are dedicated to the study of effects of scalar fields in the Standard Theory, analysis of limits on the mass of the Higgs boson, the calculation of quantum corrections to the effective potential of scalar fields and the problem that naturalness of elementary scalars poses for the structure of ultraviolet divergences of the potential.

A large part of this work is due to Nicola Cabibbo with whom we have been linked in a collaboration that lasted several decades. Nicola's contributions, several of which are on subjects not usually treated in introductory texts on gauge theories, are unmistakable for their originality and clarity.

In conclusion, we would like to express particular gratitude to Guido Altarelli, Riccardo Barbieri, John Iliopoulos, Gino Isidori, Giorgio Parisi, Antonio Polosa, Veronica Riquer, Massimo Testa, and to many other colleagues for numerous conversations and comments on the subject, and also express our affectionate thanks to Paola Cabibbo, for the patience with which she has followed the slow process of writing. Finally, we would like to acknowledge the fact that this book owes much to the comments we have received from our students, over the course of the years.

Luciano Maiani and Omar Benhar

Rome, August 2016 


\section{The Authors}

Luciano Maiani is emeritus professor of theoretical physics at the University of Rome, "La Sapienza", and author of more than two hundred scientific publications on the theoretical physics of elementary particles. Together with S. Glashow and J. Iliopoulos, Maiani made the prediction of a new family of particles, those with "charm", which form an essential part of the unified theory of the weak and electromagnetic forces. He has been president of the Italian Institute for Nuclear Physics (INFN), director-general of CERN in Geneva and president of the Italian National Council for Research (CNR). He has promoted the development of the Virgo Observatory for gravitational wave detection, the neutrino beam from CERN to Gran Sasso and at CERN has directed the crucial phases of the construction of the Large Hadron Collider (LHC). He has taught and worked in numerous foreign institutes. He was head of the theoretical physics department at the University of Rome, "La Sapienza", from 1976 to 1984 and held the chair of theoretical physics from 1984 to 2011. He is a member of the Italian Lincean Academy and a fellow of the American Physical Society. For his scientific work, he has been awarded the J. J. Sakurai Prize, the Enrico Fermi Prize, the Dirac Medal, the High Energy and Particle Physics Prize of EPS and the Bruno Pontecorvo Prize.

Omar Benhar is an INFN research director and teaches gauge theories at the University of Rome, "La Sapienza". He has worked extensively in the United States as a visiting professor at the University of Illinois and Old Dominion University, and was an associate scientist at the Thomas Jefferson National Accelerator Facility. Since 2013, he has served as an adjunct professor at the Centre for Neutrino Physics of Virginia Polytechnic Institute and State University. He is the author of more than one hundred scientific papers on the theory of many-particle systems, the structure of compact stars and electroweak interactions of nuclei.

Nicola Cabibbo (1935-2010) was professor of Theoretical Physics and Elementary Particle Physics at the Rome Universities La Sapienza and Tor Vergata, and held research and teaching positions in prestigious institutions such as Harvard University, the Institute for Advanced Studies, Princeton, CERN, Geneva, University of California at Berkeley and Université Paris VI. In 1962, Cabibbo discovered the phenomenon of quark mixing, described by a new nat- 
ural constant, the Cabibbo angle, measured with great accuracy in semileptonic weak decays of hadrons. According to a recent analysis, Cabibbo's paper on quark mixing was the most influential article published in the journals of the American Physical Society during 1893-2003. In the 1980s, Cabibbo provided important momentum to the applications of numerical techniques to theoretical physics, notably the gauge theories of strong interactions, promoting and leading the development of the family of APE (Array Processor Experiment) supercomputers. He served as a member of a number of learned societies: Accademia Nazionale dei Lincei and Accademia delle Scienze di Torino, in Italy, National Academy of Science and American Association for Art and Sciences, in the United States, and Accademia Pontificia delle Scienze, which he chaired from 1993. An internationally reputed science manager, Cabibbo was president of Istituto Nazionale di Fisica Nucleare (INFN) and of Ente Nazionale per le Nuove Tecnologie per Energia e Ambiente (ENEA). He was the recipient of the J.J. Sakurai Prize (APS), the Medaglia Matteucci (Accademia Nazionale dei XL), the Dirac Medal (ICTP Trieste), and the Benjamin Franklin Medal. 


\section{INTRODUCTION}

\section{CONTENTS}

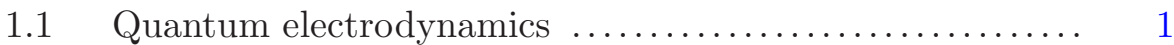

1.2 Units and other conventions. ..................... 3

\subsection{QUANTUM ELECTRODYNAMICS}

In this course we will largely focus on quantum electrodynamics, the field theory which describes the interaction of charged particles with an electric field. Quantum electrodynamics, or QED, is an incomplete theory, given that all the elementary particles are also subject to the weak interaction and, in the case of quarks, also to the strong interaction.

A more complete theory, which takes account of both electromagnetic and weak and strong interactions, is provided by the so-called Standard Theory (or Standard Model). The Standard Theory is also incomplete, given that it does not take account of gravitational interactions. ${ }^{1}$

Despite its limitations, QED is very interesting for several reasons:

- QED has a wide range of interesting physical applications, from the interactions of photons and electrons to the fine structure of matter at the atomic level.

- QED was the first field theory to have been studied in detail, mainly using the method of Feynman diagrams, to address fundamental issues such as the occurrence of infrared and ultraviolet divergences and renormalisation.

- QED is a gauge theory, and is therefore the prototype of the Standard Theory. A study of QED is a valuable introduction to the study of the Standard Theory.

${ }^{1}$ Although at the level of classical (non-quantum) physics, gravitational interactions are described very successfully by Einstein's general theory of relativity, and in many cases even Newton's theory can be satisfactorily adequate, there still does not yet exist a universally accepted quantised form of gravity. The most widely held opinion is that it will be necessary to progress from a field theory to a string theory, an argument beyond the scope of this course. 


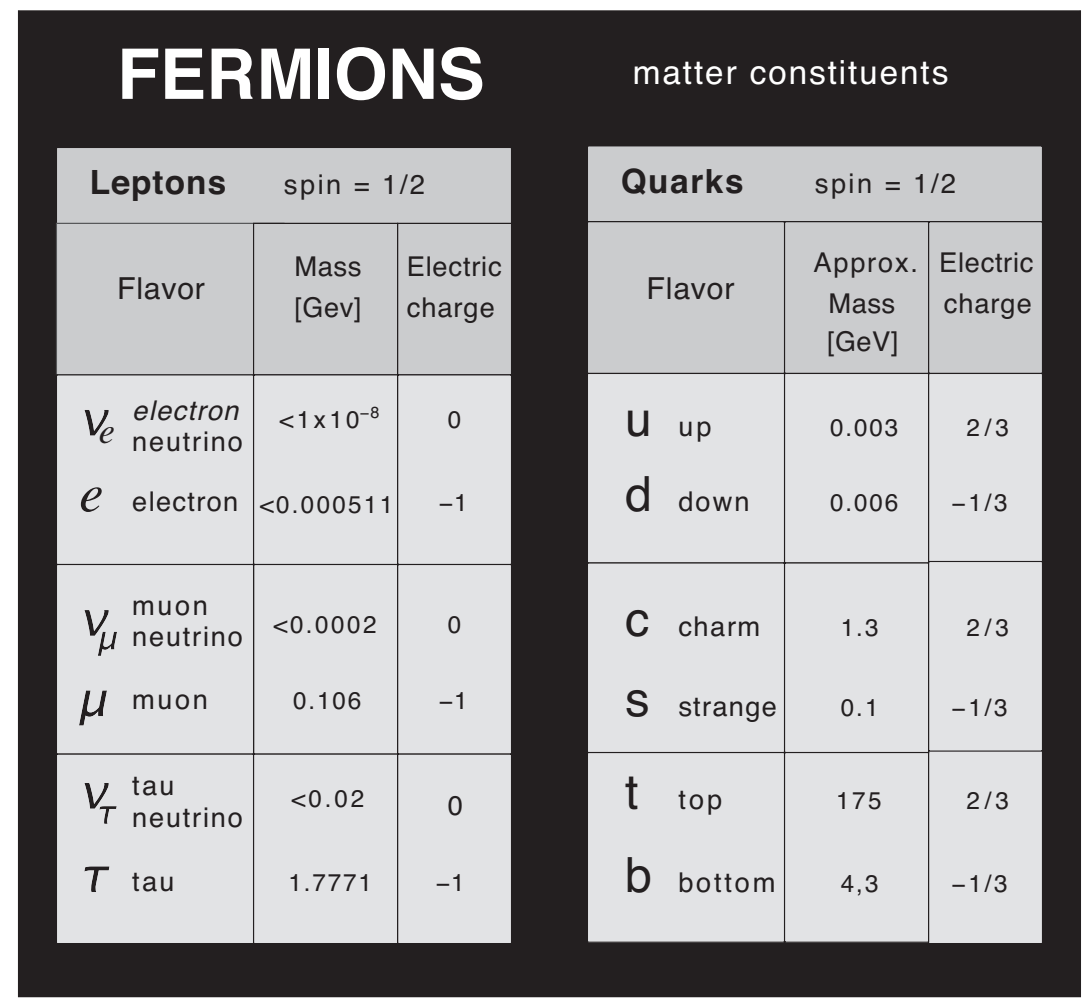

Figure 1.1 Fundamental fermions.

- Among the predictions of the Standard Theory, some of those related to QED are confirmed with the highest precision.

The most precise test of QED is currently given by the experimental value of the anomalous magnetic moment of the electron. The Dirac equation assigns to the electron a magnetic moment equal to one Bohr magneton ${ }^{2}, \frac{e}{2 m}$, but this result must be corrected because of the interaction with the radiation field by a factor $\left(1+a_{e}\right)$, where $a_{e}$ is the magnetic anomaly, which can be expressed as a power series in $\alpha$, the fine structure constant:

$$
a_{e}=\frac{\alpha}{2 \pi}+\cdots
$$

The experimental values for the electron and positron are known with

\footnotetext{
${ }^{2}$ Units and other conventions are discussed at the end of this chapter.
} 
errors of the order of 0.004 parts per million:

$$
\begin{aligned}
& a_{e^{-}}^{\operatorname{Exp}}=(1159652.1884 \pm 0.0043) \times 10^{-9}, \\
& a_{e^{+}}^{\operatorname{Exp}}=(1159652.1879 \pm 0.0043) \times 10^{-9},
\end{aligned}
$$

to be compared with the theoretical prediction:

$$
a_{e^{-}}^{\text {Th }}=(1159652.1535 \pm 0.0240) \times 10^{-9} \quad \text { (0.02 parts per million).(1.2) }
$$

The error quoted for the theoretical prediction is mainly due to the uncertainty of the value of $\alpha$, obtained from a measurement of the quantum Hall effect. The theoretical prediction is based on calculations of $a_{e}$ up to terms in $\alpha^{4}$.

In this course we will discuss quantum electrodynamics using the method of Feynman path integrals, which has been shown to be definitely superior to the more traditional method of canonical quantisation [1] to handle quantum theories characterised by gauge symmetries. The concepts developed here provide a useful foundation to discuss the Standard Theory [2].

We recall that electromagnetism is characterised by the invariance of all the observable quantities, for example the electric and magnetic fields $\mathbf{E}$ and B, with respect to transformation of the vector potentials:

$$
A_{\mu} \rightarrow A_{\mu}^{\prime}=A_{\mu}+\partial_{\mu} f
$$

where $f$ is an arbitrary function. We can consider this transformation as due to a transformation operator $U_{f}$, and write

$$
U_{f} A_{\mu} U_{f}^{-1}=A_{\mu}+\partial_{\mu} f .
$$

If we carry out two successive transformations, $U_{f}, U_{g}$, we will have

$$
U_{g} U_{f} A_{\mu} U_{f}^{-1} U_{g}^{-1}=A_{\mu}+\partial_{\mu} f+\partial_{\mu} g,
$$

and we can easily verify that the set of $U_{f}$ form a commutative (Abelian) group:

$$
U_{g} U_{f}=U_{f} U_{g}=U_{g+f} .
$$

In the case of electromagnetism, we are therefore confronted with a particularly simple type of gauge invariance, while in the Standard Theory we must deal with non-commutative (non-Abelian) gauge symmetries. Even if the Feynman path integral method is not indispensable for the quantum description of electromagnetism, it is preferred in the case of the Standard Theory.

\subsection{UNITS AND OTHER CONVENTIONS.}

Heisenberg and Schrödinger representations. In what follows, we will principally use the Heisenberg representation, where the operators have a dependence on time:

$$
O(t)=e^{i t H} O e^{-i t H} \quad, \quad O=O(0) .
$$


We will note in passing when it will be preferable to use the Schrödinger representation. In each case, operators and states which will not be shown with any explicit dependence on time, for example $O|m\rangle$, are meant to be operators and fixed states, equivalent to $O(t=0)|m\rangle$ in the Heisenberg representation and to $O|m, t=0\rangle$ in the Schrödinger representation.

Units. We will use the system of units in which $\hbar=1$ and $c=1$.

Other conventions. We will follow the conventions used in $[1,3]$ for 4vectors and the Dirac matrices. In particular, the scalar product between two 4 -vectors $p=\left\{p^{0}, \mathbf{p}\right\}$ and $q=\left\{q^{0}, \mathbf{q}\right\}$ will be denoted as $p q \equiv p^{\mu} q_{\mu}=$ $p^{0} q^{0}-\mathbf{p} \cdot \mathbf{q}$. 


\section{References}

L. Maiani and O. Benhar, Relativistic Quantum Mechanics, CRC Press, 2015.

L. Maiani , Electroweak Interactions, CRC Press, 2015.

F. Mandl and G. Shaw, Quantum Field Theory, Wiley, 1984.

R.P. Feynman and A.R. Hibbs, Quantum Mechanics and Path Integrals, McGraw-Hill, 1965.

P. A. M. Dirac, Physikalische Zeitschrift der Sowjetunion, Band 3, Heft 1 (1933), Reprinted in Quantum Electrodynamics, ed. J.

Schwinger, Dover, 1958.

H. Kleinert, Path Integrals in Quantum Mechanics, Statistics, Polymer Physics, and Financial Markets, World Scientific, Singapore, 2006.

J. J. Sakurai, Modern Quantum Mechanics, Addison-Wesley, 1994.

J. D. Bjorken and S. Drell , Relativistic Quantum Fields, McGraw-Hill, 1965.

C. Itzykson and J.-B. Zuber, Quantum Field Theory, McGraw-Hill, 1980.

L. Landau and E. Lifschitz, Mecanique Quantique. Theorie Non Relativiste, Mir, 1967.

H. Lehman, K. Symanzik, and W. Zimmermann, Nuovo Cimento 1 (1955), p. 205.

F. Bloch and A. Nordsieck, Phys. Rev. 52 (1937), p. 54.

H.A. Bethe, Phys. Rev. 72 (1947), p. 339.

G. Abbiendi, et al., OPAL Collaboration, Eur. Phys. J. C 45 (2006), p. 1.

M. Gell-Mann and F.E. Low , Phys. Rev. 95 (1954), p. 1300.

C.N. Yang and R.L. Mills, Phys. Rev. 96 (1954), p. 191.

L. Faddeev and V. Popov, Phys. Lett. B 25 (1967), p. 29.

V.N. Gribov, Nucl. Phys. B 139 (1978), p. 1.

R.P. Feynman , Acta Phys. Polon. 24 (1963), p. 697.

B.S. DeWitt , Phys. Rev. 162 (1967), p. 1195.

R.E. Cutkosky, J. Math. Phys. 1 (1960), p. 429.

M.E. Peskin and D.V. Schroeder, An Introduction to Quantum Field Theory, Perseus Books, 1995.

K.A. Olive, et al., Particle Data Group, Chin. Phys. C 38 (2014), p. 090001.

S. Bethke, Eur. Phys. J. C 64 (2009), p. 689.

J.C. Pati and A. Salam, Phys. Rev. D 10 (1974), p. 275.

H. Georgi and S.L. Glashow, Phys. Rev. Lett. 32 (1974), p. 438.

G. Ross, Grand Unified Theories The Benjamin/Cummings PublishingCo. (Menlo Park, California), 1985, see also P. Ramond, Journeys beyond the Standard Model, Perseus Books (Cambridge, Massachusetts), 1999.

S.R. Coleman and E.J. Weinberg , Phys. Rev. D 7 (1973), p. 1888.

C. Ford, D.R.T. Jones, P.W. Stephenson, and M.B. Einhorn , Nucl. Phys. B 395 (1993), p. 17.

K.G. Wilson and J.B. Kogut, Phys. Rep. 12 (1974), p. 75.

L. Maiani , G. Parisi , and R. Petronzio , Nucl. Phys. B 136 (1978), p. 115.

N. Cabibbo , L. Maiani , G. Parisi , and R. Petronzio , Nucl. Phys. B 158 (1979), p. 295.

M. Sher, Phys. Rep. 179 (1989) 273; M. Lindner, M. Sher and H. W. Zaglauer, Phys. Lett. B 228 (1989), p. 139.

G. Altarelli and G. Isidori, Phys. Lett. B. 337 (1994), pp. 141M. Sher, Phys. Lett. B 317 (1993) 159, ibidem 331 (1994).

A. Salvio and A. Strumia, JHEP 1406 (2014), p. 080.

G. Degrassi , S. Di Vita, J. Elias-Miro , J.R. Espinosa , G.F. Giudice , G. Isidori , and A. Strumia , JHEP 1208 (2012), p. 098.

T. Burnett and J.J. Levine, Phys. Lett. 24 (1967) 467; S. J. Brodsky and J. Sullivan, Phys. Rev. 156 (1967) 1644; T. Kinoshita et al, Phys. Rev. D 2 (1970), p. 910.

R. Jackiw and S. Weinberg, Phys. Rev. D 5 (1972) 2396; G. Altarelli, N. Cabibbo and L. Maiani, Phys. Lett. B 40 (1972), p. $415 ;$ I. Bars andM. Yoshimura, Phys. Rev. D6 (1972) 374; K. Fujikawa, B. W. Lee andA. I. Sanda, Phys. Rev. D6 (1972) 2923.

M. Srednicki , in Quantum Field Theory, Cambridge University Press, 2007, .

J.S. Bell and R. Jackiw, Nuovo Cim. A 60 (1969), p. 47; S. L. Adler, Phys. Rev. 177 (1969) 2426.

C. Bouchiat, J. Iliopoulos, and P. Meyer, Phys. Lett. B 38 (1972), p. 519.

A. Hoecker, W.J. Marciano, In: K. A. Olive, et al., Particle Data Group, Chin. Phys. C 38 (2014), p. 090001.

J. Schwinger, Proc. Natl. Acad. Sci. U. S.. 37 (1951), 452; 37, (1951), 455.

G. Jona-Lasinio, Nuovo Cimento 34 (1964), p. 1790.

J. Goldstone, A. Salam, and S. Weinberg , Nuovo Cimento 127 (1962), p. 965.

J. Iliopoulos, C. Itzykson, and A. Martin, Rev. Mod. Phys. 47 (1975), p. 165.

C.G. Callan, jr., Phys. Rev. D. 5 (1972), 3202;K. Symanzik, Comm. Math. Phys. 18 (1970) 227; Commun. Math. Phys. 23 (1971) 49.

G. 't Hooft , Naturalness, Chiral Symmetry and Spontaneous Chiral Symmetry Breaking, in Recent Developments in Gauge Theories, Plenum Press, 1978, previously, similar considerations were discussed in K. G. Wilson, Phys. Rev. D 3 (1971) 1818.

J. Wess and B. Zumino, Nucl. Phys. B70 (1974) 39; V. P. Akulov, D. V. Volkov, Theor. Math. Phys. 18 (1974), p. 28.

L. Maiani, in Proc. of the Summer School on Particle Physics, Gif-sur-Yvette, 3-7 September 1979, Ed. M. Davier et al., IN2P3, Paris, 1979; M. Veltman, Acta Phys. Polon. B12 (1981) 437; E. Witten, Nucl. Phys. B 188 (1981) 513 and Phys. Lett. B 105 (1981) 267.

P. Fayet, Nucl. Phys. B. 90 (1975), 104; Phys. Lett. B 64, 159 (1976); B 69, 489 (1977).

S. Dimopoulos and H. Georgi, Nucl. Phys. B 193 (1981), p. 150.

J. Gunion, H. Haber, G. Kane, and S. Dawson, The Higgs Hunter's Guide, Reading, 1990; S. Heinemeyer, W. Hollik and G. Weiglein, Phys. Rep. 425265 (2006); A. Djouadi, Phys. Rep. 459 (2008), p. 1.

K. Agashe, R. Contino, and A. Pomarol, Nucl. Phys. B 719 (2005), p. 165.

M. Abramowitz and I.A. Stegun, Handbook of Mathematical Functions, Dover, Lonond, 1972.

H. Jeffreys and M. Jeffreys, Methods of Mathematical Physics, Cambridge University Press, Lonond, 1972. 
